\title{
Pathologie des blessures mettant à nu le bois chez les végétaux ligneux
}

\author{
C Grosclaude \\ INRA, station de Pathologie végétale, BP 94, F 84143 Montfavet cedex, France
}

(Reçu le 31 août 1992; accepté le 22 décembre 1992)

\begin{abstract}
Résumé - Les blessures qui mettent le bois à nu ont des origines diverses, accidentelles le plus souvent, parfois naturelles (élagage naturel). Les blessures ont pour conséquence la rupture d'un certain nombre d'éléments conducteurs dans lesquels la sève brute est en général sous tension. Toute blessure détermine donc un retrait de la sève et une aspiration de l'air atmosphérique et des spores de micro-organismes qu'il contient ; par la suite, on observe la formation de thylles et de gommes. Les spores des micro-organismes aspirées dans les éléments conducteurs peuvent pénétrer à plusieurs centimètres de profondeur, mais le bois joue néanmoins un rôle de filtre du fait notamment de la longueur limitée des vaisseaux et des éléments qui les forment. Les conditions qui règnent dans le bois (humidité, teneur en oxygène et en substances toxiques, etc) opèrent une sélection parmi les micro-organismes présents. Ceux qui se développent peuvent provoquer des pourritures du bois ("carie») dues à la dégradation de la lignine ou/et de la cellulose des membranes (champignons lignivores). Les premiers organismes qui se développent dans le bois sont des pionniers, mais une succession parasitaire s'établit par la suite, selon le pouvoir de compétitivité de chaque organisme. Dans l'arbre, en réponse aux agressions subies, on peut mettre en évidence une zone de réaction et une zone de bois dégradé, chacune ayant des caractéristiques bien différentes. Le concept de compartimentation selon Shigo (CODIT) est exposé. Enfin, les possibilités de protection à l'égard des micro-organismes lignivores sont discutées, aucune méthode de lutte n'étant totalement satisfaisante.
\end{abstract}

blessures / tissu ligneux / champignon lignivore / altération du bois / succession parasitaire / compartimentation

Summary - Pathological study of exposed wood wounds in woody plants. Wounds which expose wood to atmospheric agents have various accidental or natural origins. All wounds result in the breakdown of several conducting elements: in these elements, the sap is under negative pressure. Therefore wounds result in the withdrawal of the sap and penetration of air as well as microorganic spores. Thyllose or gummosis subsequently occurs. The penetration of microorganic spores can reach several centimeters in depth, but the wood neverthless acts as a filter due to the limited length of the vessels and vessel elements. Conditions in the wood including humidity, oxygen and extractive content put a selection pressure on the microorganisms present. Those which develop can induce wood decay ("carie") by degradation of lignin and/ or cellulose in the membranes (wood-destroying fungi). The first microorganisms to become established are considered pioneers, but thereafter a succession of microorganisms occurs according to the competitive ability of each. In the tree, the response to attack is the formation of a reaction zone (discolored wood) and a zone of wood decay. The concept of compartmentalization according to Shigo (CODIT) is explained. Finally, possibilities for control of wood-destroying fungi are discussed, but no method has been found fully satisfactory.

wound / woody tissue / wood destroying fungus / wood decay / microorganic succession / compartmentalization 


\section{INTRODUCTION}

Comme tous les êtres vivants, les végétaux ligneux sont amenés au cours de leur existence à subir des blessures diverses; si l'on excepte les blessures superficielles qui n'intéressent que les tissus corticaux, la plupart d'entre elles ont pour conséquence la mise à nu du bois, qui se trouve ainsi exposé aux agents extérieurs. De telles blessures ont des origines diverses, qu'it s'agisse de celles provoquées volontairement par l'homme au cours des opérations de taille, d'émondage, d'élagage ou encore de celles provoquées accidentellement par les intempéries (bris de branches), par l'homme ou les animaux (rongeurs, etc). Mais il faut aussi considérer les blessures naturelles comme celles provoquées par la chute des feuilles (qui met à nu un ou plusieurs faisceaux libéro-ligneux) ou encore l'élagage naturel, qui concerne les branches peu éclairées, mal alimentées, et qui se réalise spontanément.

Si nous excluons de notre propos les blessures du système racinaire que peuvent éventuellement provoquer des insectes ou des nématodes, ou encore celles qui résultent d'opérations, fréquentes en milieu urbain (creusement de tranchées, etc), on peut dire que, quelle que soit l'origine des blessures, celles-ci vont exposer le tissu ligneux à l'air libre : il en découlera un certain nombre de conséquences importantes sur le plan phytosanitaire, conséquences qui font l'objet de cette étude et qui seront examinées à la lumière des données bibliographiques en notre possession.

\section{LES TISSUS LIGNEUX EXPOSÉS}

Les blessures, dues à la section des branches d'un arbre par exemple, mettront à nu le bois, qui est l'ensemble des tissus assurant la conduction de la sève et le soutien du végétal. La conduction est assurée par les éléments conducteurs (trachéides chez les gymnospermes, vaisseaux chez les angiospermes, mais aussi, chez ces derniers, fibres libriformes, fibres-trachéides) de la partie la plus externe du bois, appelée aubier (fig 1). Si tout le corps ligneux possède par lui-même un rôle de soutien, ce rôle est le seul qu'assure la partie centrale, non fonctionnelle, appelée cœur.

Le cœur s'élabore progressivement aux dépens de l'aubier, et sa formation ne commence

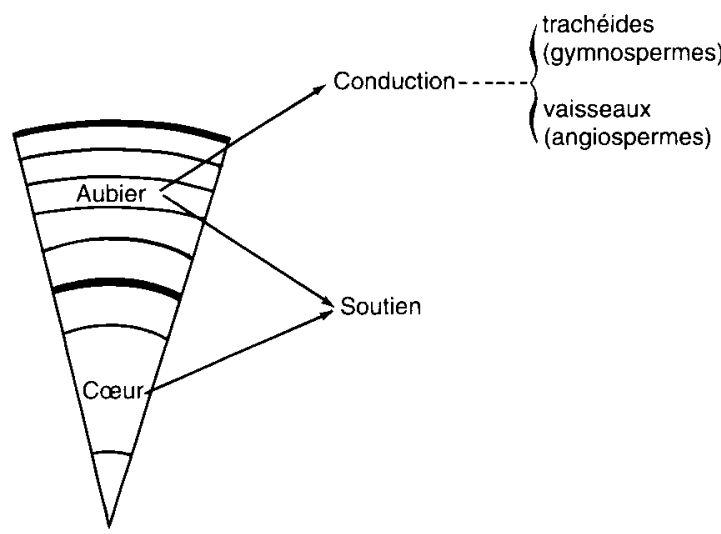

Fig 1. Les éléments conducteurs.

qu'au bout d'un certain temps, plus ou moins long, très variable selon les espèces (2 à 200 ans) : de ce fait, la section d'une branche pourra, selon son âge, exposer ou non le bois de cœur. Toutefois, le cœur, une fois formé et possédant toutes ses caractéristiques physiologiques propres, n'est visible que chez certaines espèces (robinier, noyer, chêne, châtaignier, pin, orme, frêne, mélèze, if, merisier) où il prend une couleur foncée due à l'accumulation de substances phénoliques (on parle alors de duramen), mais il ne faut pas confondre le cœur, lorsqu'il est coloré, avec diverses altérations de l'aubier, d'origine pathologique que nous étudierons plus loin. Chez d'autres espèces (hêtre, érable, sapin, épicéa, charme, cèdre), le cœur est peu ou pas - visible. Visible ou non, il s'agit dans bien des cas d'un tissu plus pauvre en eau que l'aubier (Mer, 1887 ; Rameau et al, 1989).

\section{CONSÉQUENCES DE LA MISE EN CONTACT DU TISSU LIGNEUX AVEC L'ATMOSPHĖRE}

Ce qui suit ne concerne pas les blessures consécutives à l'élagage naturel, dont les conséquences seront envisagées plus loin.

Vaisseaux ou trachéides sont les éléments conducteurs de la sève brute ascendante, et la rupture de ces éléments dont l'ensemble est, en première approximation, assimilable sur le plan physique à une colonne de liquide, aura un effet différent selon la pression qui y règne. Si la pression est positive, il y aura écoulement de liquide : tel est le cas au printemps et à la fin de l'automne ; le phénomène est mis à profit dans certaines contrées lorsque l'on veut récolter la sève de bouleaux ou d'érables. C'est ce même phénomène qu'observe le viticulteur lorsque sa vigne, une fois taillée, "pleure», mais il s'agit toujours 
d'un phénomène fugace : en règle générale, il règne dans le bois de l'arbre, même en hiver, une pression négative, la colonne de liquide étant dite sous tension (Dixon, 1924 ; Huguet, 1973 ; Zimmermann et Brown, 1974), cet état de tension étant relativement instable et apte à engendrer des phénomènes de cavitation et d'embolie (Cruiziat et Tyree, 1990) - en revanche, la pression est toujours positive dans le phloème, où circule la sève élaborée, descendante. On constate donc, lors de la section d'une branche, le retrait de la sève dans les éléments conducteurs et le remplacement de celle-ci par de l'air: autrement dit, toute blessure va être le siège d'une aspiration de l'air atmosphérique et de tout ce qu'il peut véhiculer, et notamment les spores de micro-organismes - champignons ou bactéries -, inévitablement présents dans l'air ou l'eau des précipitations. Les blessures, du fait de l'aspiration dont elles sont le siège, constituent donc pour les micro-organismes des portes d'entrée privilégiées, sinon indispensables : en effet, si après avoir été déposées à la surface d'une blessure elles y demeuraient, les spores de bien des espèces ne pourraient résister à une dessiccation trop intense ni à une exposition trop prolongée à la lumière extérieure. Cette aspiration peut être mise en évidence par des expériences très simples : au niveau d'une coupe, elle concerne aussi bien la partie distale que la partie proximale et elle n'est que peu influencée par la force de la pesanteur (figs 2 et 3 ). Importante au moment de la coupe, même pendant le repos végétatif, cette aspiration diminue rapidement avec le temps (Grosclaude, 1974). D'une façon générale, la mise en contact du tissu ligneux avec l'atmosphère aura en outre pour conséquence une déshydratation des tissus, plus ou moins importante et plus ou moins persistante selon les conditions météorologiques du moment. Par la suite, chez les angiospermes uniquement, comme on peut le montrer expérimentalement, la perte d'eau au niveau des vaisseaux va provoquer chez ceux-ci la formation de thylles ou de gommes.

La formation de thylles, ou thyllose, résulte de l'intrusion, dans la lumière d'un vaisseau au niveau d'une ponctuation, de la lamelle moyenne de la paroi de ce vaisseau et du contenu d'une cellule associée (cellule du parenchyme jouxtant les vaisseaux, et surtout rayons ligneux) ; cette intrusion par les orifices d'une ponctuation forme ainsi une hernie à l'intérieur du vaisseau (fig 4). Mais il est nécessaire pour cela que les ponctuations aient un diamètre d'au moins 10 microns (il ne s'agit pas ici du diamètre des pores des ponc- tuations qui, eux, sont beaucoup plus petits). $\mathrm{Ce}$ cas se produit entre autres chez le châtaignier, le hêtre, le platane, le robinier, etc.

La formation de gommes ou gommose, résulte d'un processus analogue; cependant il s'agit non d'une intrusion d'une partie des cellules associées mais d'une sécrétion interne qui se déverse dans les vaisseaux par les ponctuations. Dans les premiers stades, on parle, parfois, non de gommes mais de gels vasculaires (Vandermolen et al, 1977). La gommose remplace la thyllose chez les espèces dont les ponc-
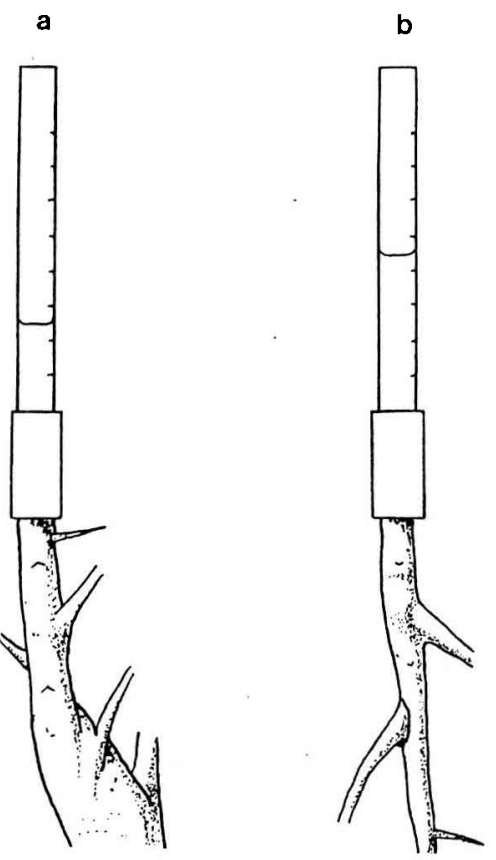

Fig 2. (D'après Grosclaude, 1974). a. Si l'on adapte un tube gradué rempli d'eau à l'extrémité d'une branche qui vient d'être sectionnée, on constate une absorption rapide du liquide. b. Avec un dispositif analogue, on constate que la partie distale absorbe également le liquide, mais en moindre quantité qu'en a. Ces expériences peuvent être réalisées lorsqu'il n'y a plus de feuilles, durant le repos végétatif.

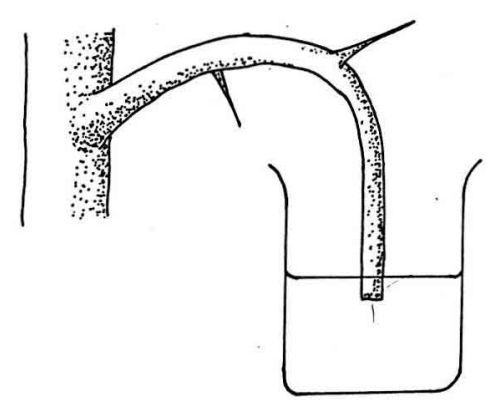

Fig 3. (D'après Grosclaude, 1974). Une branche fraîchement sectionnée est courbée de façon à ce que l'extrémité plonge dans une solution colorée. Peu de temps après, on peut constater, en refendant la branche, qu'une certaine quantité de liquide a été aspirée. 


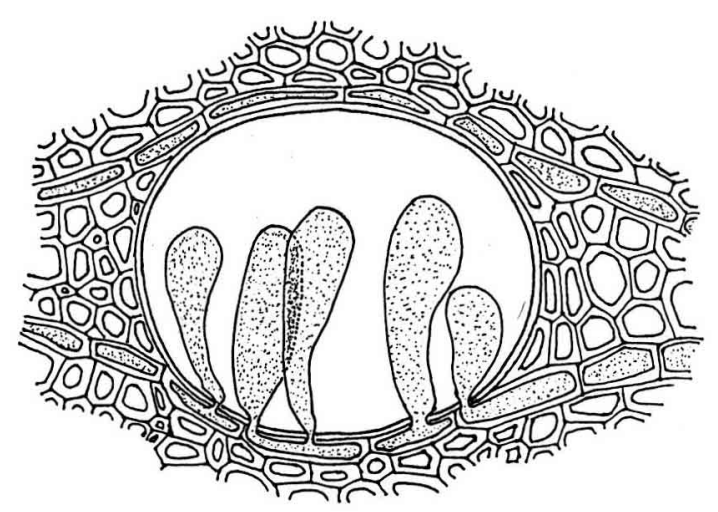

Fig 4. Schéma montrant l'obstruction partielle d'un vaisseau par des thylles (d'après Chattaway, 1949).

tuations ont une dimension inférieure à $10 \mathrm{mi}-$ crons, par exemple, les Rosacées fruitières, les érables, le tilleul, le marronnier, etc (Chattaway, 1949 ; Catesson et al, 1976 ; Bonsen et Bucher, 1991).

Thyllose et gommose s'élaborent uniquement aux dépens des cellules vivantes, c'est-à-dire celles de l'aubier jusqu'à la zone de transition avec le duramen où, une fois présentes, elles persistent. Si c'est peut-être à tort que thyllose et gommose ont été attribuées en premier lieu à l'invasion par des micro-organismes, elles sont considérées avec raison comme des réactions de défense de l'arbre aux agressions puisqu'elles peuvent obturer partiellement ou même complètement les vaisseaux, bloquant ainsi non seulement l'entrée d'air, mais aussi le transport des spores ou des toxines des agents pathogènes (et la sève elle-même). À ce titre, comme nous le verrons plus loin, elles sont un des éléments constitutifs des cloisons de "compartimentation".

\section{LA PÉNÉTRATION DES MICRO- ORGANISMES À L'INTÉRIEUR DU BOIS}

Les spores présentes dans l'atmosphère, soit libres, soit en suspension dans l'eau des précipitations, ou bien encore déposées à la surface de la blessure par les outils de taille, vont être immédiatement aspirées dans les éléments conducteurs. En effet, les dimensions des spores sont en général beaucoup plus faibles que le diamètre des vaisseaux, qui varie de 16 à $160 \mu$ dans les bois à pores diffus et de 60 à $800 \mu$ dans les bois à zone poreuse.

Le mot "pore" étant un terme commode utilisé dans la pratique pour désigner la section transversale d'un vaisseau ou d'une trachéide vasculaire, on définit ainsi les deux types de bois évoqués ci-dessus :

- bois à pores diffus: bois dans lequel les cernes présentent des pores de taille et de répartition relativement uniforme ou avec des modifications progressives.

- bois à zone poreuse: bois dans lequel les pores du bois initial (bois de printemps) sont manifestement plus gros que ceux du bois final (bois d'été) et forment une zone ou un anneau bien marqué (anonyme, 1964).

La profondeur de pénétration de ces spores à l'intérieur du bois est très variable, car elle dépend de l'âge de la plaie (la vitesse d'aspiration au niveau d'une plaie décroît avec l'âge et finit par s'annuler chez une plaie plus âgée), mais surtout de la longueur limitée des trachéides ou des vaisseaux (Beckman et Keller, 1977). Cette profondeur de pénétration peut atteindre plusieurs dizaines de centimètres chez certains feuillus dont les vaisseaux ont souvent une longueur importante (elle peut atteindre une longueur de $10 \mathrm{~m}$ chez le chêne sessile, par exemple). Mais il faut préciser que les spores non germées ne peuvent traverser la paroi d'un vaisseau, les pores des membranes des ponctuations qui tapissent cette paroi ayant un diamètre compris entre 10 et 100 nanomètres environ (fig 5); cette longueur des éléments conducteurs est en revanche beaucoup plus réduite chez les conifères dont les trachéides mesurent au plus 5 à $10 \mathrm{~mm}$ (Hintikka, 1973). Au niveau des "nœuds", c'est-à-dire des bifurcations des branches, un rétrécissement des vaisseaux peut exister ; de plus, en de tels endroits, on a montré que les extrémités des vaisseaux se trouvaient en beaucoup plus grand nombre qu'ailleurs (Salleo et al, 1984) : ce sont donc de véritables goulots d'étranglement qui se constituent dans ces localisations. De plus, les vaisseaux, étant constitués eux-mêmes par des éléments de vaisseaux plus courts, associés bout à bout, et munis aux extrémités de perforations plus ou moins ouvertes (improprement appelées parfois "pores") et d'appendices de forme variable, ne sont donc pas de simples tubes aux parois lisses : on ne peut alors en toute rigueur leur appliquer les lois de Poiseuille applicables seulement à des tubes capillaires aux parois parfaitement lisses. Les spores rencontrent donc un certain nombre d'obstacles sur leur passage et cela, ajouté à la longueur limitée des vaisseaux, fait qu'en pratique le bois joue le rôle d'une sorte 


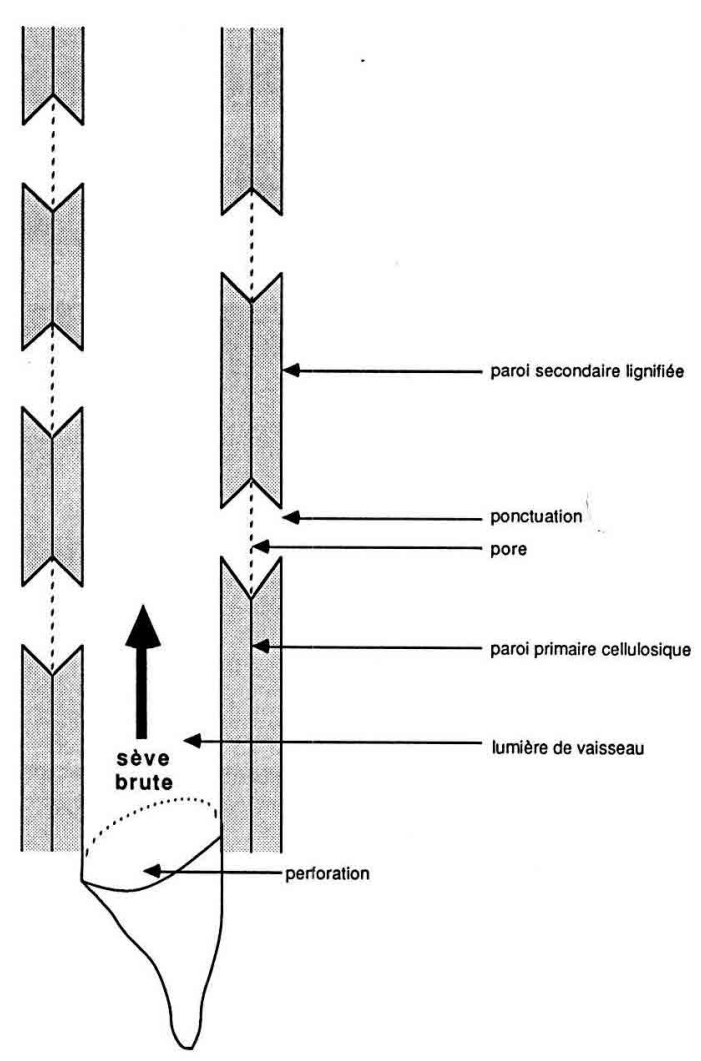

Fig 5. Coupe schématique d'un élément de vaisseau.

de filtre, la quantité de spores déposées dans les éléments conducteurs étant d'autant plus faible que I'on s'éloigne de la surface de coupe (Grosclaude, 1974 ; Zimmermann, 1983) (tableau I). Ainsi, on a pu montrer que les spores d'Eutypa lata, parasite de la vigne, après avoir pénétré dans les vaisseaux, s'accumulaient au niveau des obstacles qui s'y trouvent, constituant alors localement l'origine de veritables foyers d'infection (Paillassa et al, 1992), mais à l'inverse, on a observé aussi que, chez la vigne, les spores

Tableau I. Effet filtre du bois de prunier sur une suspension de spores de Chondrostereum purpureum (d'après Grosclaude, 1974).
Longueur du fragment de tige : $\mathrm{cm}$
Titre de la suspension recueillie : spores $/ \mathrm{ml}$

$\begin{array}{rr}0 & 2300000 \\ 5 & 1260000 \\ 10 & 510000 \\ 20 & 44000 \\ 25 & 28000\end{array}$

ayant pénétré dans les vaisseaux pouvaient être rejetées à l'extérieur durant la courte phase des «pleurs» qui résulte de l'établissement d'une pression positive dans les éléments conducteurs (Paillassa et al, 1992).

Une fois que les spores ont ainsi pénétré, celles-ci, si elles trouvent des conditions de milieu favorables, pourront germer puis donner naissance à des colonies qui, progressivement, se développeront dans le tissu ligneux. Or, précisément, il est important de considérer que le retrait de la sève, consécutif à la blessure, et son remplacement par de l'air dans les éléments conducteurs, constituent un facteur favorable pour les micro-organismes qui n'auraient pu se développer dans les conditions d'anoxie régnant habituellement dans l'aubier sain.

\section{L'ÉLAGAGE NATUREL : UN CAS PARTICULIER}

L'élagage naturel est le processus par lequel les branches les moins bien alimentées - donc les moins vigoureuses - et les moins bien éclairées vont être éliminées, permettant ainsi, entre autres, la constitution d'un tronc rectiligne exempt de branches latérales. Deux mécanismes bien différents permettent la réalisation de l'élagage naturel :

- L'existence, à la base des branches, d'une zone d'abscission quelque peu semblable à celle qui se forme dans le pétiole des feuilies avant leur chute, et qui est constituée essentiellement d'un parenchyme peu vascularisé et sans fibres lignifiées. Cette zone est par la suite recouverte ou non par un tissu ligneux qui consolidera la branche; si ce recouvrement ne se produit pas, l'abscission aura lieu. Tel est le cas du Populus serotina (Thomas, 1933) ainsi que d'autres espèces de ce genre et des genres Quercus, Salix, Betula, Celtis, Fagus, Ulmus (Christensen, 1975 ; Chaney, 1979). Sous l'action du vent ou d'autres contraintes externes, les branches possédant cette zone vont chuter avant d'avoir séché et portant encore des feuilles, créant ainsi une blessure naturelle plus ou moins importante; ce processus a reçu le nom de cladoptose (de clados, rameau et "-ptose», chute), tandis que, chez le peuplier, on parle de décurtation.

- Dans d'autres cas, notamment chez le platane, les branches mal alimentées se dessèchent, meurent et sont envahies par une myco- 
flore variée spécifique de l'hôte. La chute de ces branches se produit alors grâce à l'action des micro-organismes présents; les blessures qui en résultent constituent un cas à part. En effet, puisqu'il s'agit de bois mort depuis un certain temps, ce bois étant vide de sève, il ne peut $y$ avoir d'aspiration par la blessure. De plus la mycoflore installée dans ces organes peut, en dépit des barrières de protection élaborées à leur base - terpènes, cônes de résine - (von Aufsess, 1975 ; Butin et Kowalski, 1990), gagner le tronc ou les branches qui les portent et s'y développer (Butin et Kowalski, 1983, 1986). Par ailleurs, on a pu mettre en évidence - chez le platane ainsi que chez le tremble (Wall et Kuntz, 1964) - un pouvoir fongistatique important des branches mortes ; l'origine de ce pouvoir fongistatique, qui existe vraisemblablement chez les autres espèces, n'est pas connue (réaction physiologique de l'arbre, produits du métabolisme des micro-organismes présents ?). Or ce pouvoir fongistatique ne s'exerce pas de la même manière sur toutes les espèces fongiques : certaines $y$ sont très sensibles, d'autres non, d'autres encore seraient favorisées par les extraits de branches mortes. Ces branches mortes, ainsi que les moignons qui subsisteront à l'intérieur du bois pour constituer plus tard les nœuds, se comportent donc comme des filtres sélectifs à l'égard des micro-organismes qui, ultérieurement, vont tenter de pénétrer par les blessures formées (Anselmi, 1990).

\section{DIVERSITÉ DES ESPÈCES PRÉSENTES DANS LE BOIS DES ARBRES. INFLUENCE DU MILIEU INTERNE SUR LEUR DÉVELOPPEMENT}

Si l'on excepte le cas de l'élagage naturel, n'importe quel micro-organisme étant susceptible de pénétrer dans un arbre à la faveur de blessures diverses, une sélection s'opère très rapidement dès la germination des spores, compte tenu des conditions particulières régnant dans le bois. Cependant, en dépit de cette sélection, l'examen microbiologique d'un arbre vivant, apparemment sain, révèle la présence de nombreux microorganismes, plus ou moins proches des blessures visibles et notamment de celles provoquées par la taille (mais il faut garder présent à l'esprit qu'il existe toujours sur un arbre de nombreuses blessures naturelles ou accidentelles peu visibles, constituant autant de portes d'en- trée pour les micro-organismes). Les microorganismes, champignons ou bactéries, qui vivent dans le bois et $s^{\prime} y$ développent sont qualifiés de lignicoles. L'état du bois et sa composition à un moment donné influeront sur le développement de ces micro-organismes, favorisant les uns ou inhibant les autres, selon les exigences propres de chacun d'entre eux. Les facteurs les plus importants pouvant influer sur le développement des micro-organismes lignicoles sont mentionnés ci-dessous.

\section{Le taux d'humidité du bois et l'aération}

Une teneur élevée en eau telle que celle existant dans l'aubier peut créer des conditions anoxiques défavorables à la plupart des microorganismes (ce qui, entre autres, justifie l'arrosage des stocks de bois abattus). À l'inverse, un trop faible taux d'humidité peut gêner la croissance des micro-organismes; toutefois, sur des arbres en place, cette éventualité se présente rarement, sinon au voisinage d'une surface de coupe. On peut alors penser que les spores simplement déposées à la surface d'une blessure et non aspirées - auront, de ce fait, peu de chances de trouver des conditions favorables à leur germination.

En règle générale, le cœur est moins riche en eau que l'aubier ; cela est vrai pour les gymnospermes, mais, chez les angiospermes, cette règle souffre de nombreuses exceptions. Ainsi, l'aubier de peuplier est moins riche en eau que le cœur (Nepveu, 1991); mais si la teneur en eau diminue, cette dernière fait place à une phase gazeuse souvent enrichie en $\mathrm{CO}_{2}$ : des taux de 10 à $20 \%$ de ce gaz, susceptibles d'arrêter la croissance de nombreuses espèces, ont parfois été observés.

\section{La teneur du bois en substances toxiques pour les micro-organismes}

Le bois contient une grande variété de substances antiseptiques, groupées sous la dénomination d'“extractives" selon la terminologie anglo-saxonne, car la résistance à la dégradation fongique que de telles substances confèrent au bois disparaît en grande partie à la suite d'une extraction par l'eau ou les solvants organiques. Les plus connues de ces substances sont des dérivés phénoliques: terpènes, flavonoïdes 
(quercétine, robinètine), tropolones (thuyaplicines), stilbènes (pinosylvine) ou tanins (Scheffer et Cowling, 1966). Ces substances, localisées surtout dans le bois de cœur et plus précisément dans la partie périphérique de celui-ci, se forment aussi dans l'aubier en réaction aux agressions de toute nature. La concentration et la nature de ces substances sont évidemment très variables selon les essences, et, de plus, il existe parmi les champignons des espèces tolérantes à l'égard de ces composés (Boddy et Rayner, 1983 ; Rayner, 1985).

\section{ACTION DES CHAMPIGNONS LIGNICOLES SUR L'ASPECT DU BOIS ET SA TEXTURE}

La présence de champignons lignicoles dans le bois est à l'origine d'altérations de divers types.

\section{Des colorations anormales}

L'envahissement du bois par des champignons lignicoles se traduit rapidement par l'apparition de colorations anormales. II s'agit principalement de zones colorées en brun plus ou moins foncé ou même en noir. On ne confondra pas ces colorations brunes d'origine pathologique avec celles résultant de la duraminisation normale du bois central. Mais on peut aussi observer des bleuissements du bois, chez les résineux notamment (dus à des espèces diverses, parmi lesquelles Ceratocystis sp, Cladosporium herbarum, Pullularia pullulans). Les champignons responsables de ces bleuissements s'attaquent au contenu des cellules, vivantes en général, et sont de ce fait de véritables parasites.

L'opposition classique entre «parasites» et "saprophytes" doit ici être nuancée, bien des espèces parasites ayant une phase de vie saprophytique. De plus, le terme de parasite, souvent considéré à tort comme synonyme de pathogène, est ambigu, certaines espèces étant pathogènes sur un hôte et non pathogènes sur un autre. Par ailleurs, les dénominations de "parasite secondaire", "parasite de faiblesse", trop imprécises ou ambiguës, devraient être abandonnées. En effet, un micro-organisme peut, dans une succession, intervenir en seconde position après des pionniers (voir plus loin), sans pour autant avoir une importance secondaire dans le processus pathologique engagé. D'autre part, on baptise souvent «parasite de faiblesse" des micro-organismes dont on ne perçoit les dégâts (et les fructifications) que sur des arbres âgés. Ces derniers sont-ils faibles pour autant, et comment définir alors la faiblesse ? De plus, dans le cas des lignivores, les dégâts observés sur des sujets âgés ont été, en général, initiés de nombreuses années auparavant. II est vrai toutefois, qu'à partir d'un certain âge la croissance de l'arbre, se ralentissant, permettra aux champignons installés dans le bois central de gagner du terrain sur l'aubier fonctionnel ; ce dernier se réduisant de plus en plus, un effet pathogène se manifestera alors, provoquant cette fois un véritable affaiblissement pouvant aboutir à la mort.

\section{Des pourritures ou "caries" du bois}

Le bois perd alors sa texture habituelle et devient friable, cassant ou pulvérulent. Les agents de pourriture du bois sont le plus souvent des basidiomycètes supérieurs ou hyménomycètes (aphyllophorales et agaricales), parfois aussi des ascomycètes (Xylaria, Daldinia, Ustulina). Ceux-ci s'attaquent aux parois cellulaires qui sont composées essentiellement de lignine et de cellulose en proportions variables.

Selon le mode de dégradation de ces constituants, on distinguera :

- les pourritures brunes (dues entre autres à Laetiporus sulphureus, Ungulina $s p$, Fistulina hepatica) sous l'action desquelles le bois, coloré en brun à brun rougeâtre, acquiert une texture cubique puis se transforme progressivement en une masse pulvérulente brune. Cela est le résultat de la dégradation de la cellulose et des hémicelluloses, laissant subsister - quoique modifiée - la lignine ;

- les pourritures blanches, les plus nombreuses, dans lesquelles le bois prend une couleur claire et une texture fibreuse (dues entre autres à Bjerkandera adusta, Trametes versicolor, Schizophyllum commune). Cette texture fibreuse concerne soit le corps ligneux dans son ensemble, soit des poches isolées : on parle alors de pourriture alvéolaire (Ungulina annosa, Xanthochrous pini) ou de pourriture tubulaire (Stereum gausapatum).

Parmi les agents de pourritures blanches, on distingue depuis Hartig (Hartig, 1878), dont les travaux, datant de la fin du siècle dernier, ont été récemment redécouverts et confirmés par la microscopie électronique : 
- des champignons qui délignifient sélectivement le bois, en s'attachant préférentiellement à la lignine de la lamelle moyenne;

- des champignons qui dégradent simultanément la lignine et les polysaccharides des membranes, provoquant l'érosion de toutes les couches des parois cellulaires (Blanchette, 1991).

Les agents de pourriture du bois sont dits lignivores. Vivant aux dépens de tissus morts complètement ou en grande partie, ce sont donc des saprophytes au sens strict. Ils sont cependant capables d'exercer un effet pathogène au sens large, puisqu'ils altèrent la qualité du bois et, de plus, compromettent gravement la résistance mécanique des arbres aux intempéries.

\section{SUCCESSIONS, COMMUNAUTÉS DE MICRO-ORGANISMES, INFECTION LATENTE}

Les pourritures internes du bois dont il vient d'être question ne sont pas, en règle générale, le résultat de l'action d'un seul micro-organisme. La pourriture du bois constitue en effet le terme d'une série d'événements dont l'origine est la mise à nu du tissu ligneux consécutive à une blessure. Le bois sain ainsi exposé est en premier lieu colonisé par des envahisseurs primaires ou pionniers; parmi ces pionniers figurent fréquemment des bactéries, mais des champignons sont également au nombre de ceux-ci. Ces pionniers peuvent parfois être de redoutables agents pathogènes comme c'est le cas de Ceratocystis fimbriata chez le platane, d'Eutypa lata chez la vigne, ou de Chondrostereum purpureum chez les arbres fruitiers. Par la suite, le bois, du fait de la présence et du développement de ces organismes pionniers, qu'ils soient pathogènes ou non, va subir des altérations importantes (changement de couleur, modifications du pH, de la teneur en eau, de la composition chimique, etc). Ces modifications influeront sur le développement d'autres microorganismes, permettant ou même favorisant leur installation dans des tissus désormais préconditionnés. On peut alors imaginer que ces microorganismes vont à leur tour modifier le substrat, permettant ainsi le développement d'une nouvelle vague d'envahisseurs (envahisseurs séquentiels), les suivants éliminant les précédents, la décomposition du bois se poursuivant alors jusqu'à ce qu'il ne reste plus rien ou presque du tissu ligneux (arbre creux). Si les premiers enva- hisseurs séquentiels sont d'abord, le plus souvent, des champignons basidiomycètes lignivores, on trouve par la suite des microorganismes très divers, champignons non basidiomycètes, bactéries, etc.

$\mathrm{Si}$ les différents micro-organismes se succèdent les uns aux autres, chacun remplaçant le précédent, on parle de succession parasitaire. Bien qu'envisagée dès 1939 par D'Aeth (D'Aeth, 1939) pour des situations particulières, la notion de succession parasitaire est relativement nouvelle en phytopathologie (du moins lorsqu'il ne s'agit pas de l'envahissement, par un cortège de saprophytes, d'un organe mort à la suite d'une attaque fongique). En effet, il fallut attendre 1967 pour que fût établi le fait que la succession parasitaire était la règle générale dans les processus de dégradation du bois par les organismes fongiques (Shigo, 1967). Cette notion, en effet, n'était pas familière aux phytopathologistes, plus habitués à assigner à une maladie bien définie un et un seul micro-organisme responsable.

C'est ainsi que la maladie de l'esca de la vigne, qui se traduit notamment par un dépérissement apoplectique des pieds atteints, accompagné d'une carie du cep, fut d'abord attribuée à l'action pathogène du seul Polyporus igniarius par Ravaz (Ravaz, 1909). Par la suite, d'autres champignons basidiomycètes furent mis en cause, et en particulier Stereum hirsutum, sans que jamais on ne pût reproduire les symptômes de la maladie par l'inoculation de l'un ou l'autre de ces champignons : on croyait pouvoir, à l'époque, attribuer à l'une ou à l'autre de ces espèces la responsabilité de la maladie, à la suite de l'observation fréquente de leur carpophores sur les ceps atteints, ou encore à leur présence décelée dans le bois carié. Or des travaux récents montrent que les symptômes de l'esca sont le résultat d'une succession parasitaire selon plusieurs voies possibles : après l'invasion du bois par des pionniers (Phialophora parasitica et Cephalosporium sp pour l'une des voies, Eutypa lata pour une seconde voie), se développent les basidiomycètes Phellinus sp ou Stereum hirsutum. Ces derniers sont incapables de se développer dans le bois sain, mais il s'installent dans les zones nécrosées par les pionniers et dégradent alors le bois qui se transforme en carie centrale ou sectorielle selon qu'il s'agit de la première ou de la seconde des voies (Larignon, 1991). Mais, d'une façon générale, ajoutons toutefois que la réalité n'est pas toujours aussi simple qu'il pourrait paraître : l'expérience nous montre en effet que, dans un même arbre, 
peuvent coexister de nombreux microorganismes imbriqués ou juxtaposés, plus ou moins proches les uns des autres, chacun occupant un volume donné (ce qui s'explique aisément du fait de la multiplicité des blessuresportes d'entrée survenant à n'importe quel endroit et tout au cours de la vie l'arbre). Si donc succession il y a, ce peut être une succession de communautés fongiques et non d'individus ou d'espèces. Entre les divers partenaires qui composent ces communautés, existent des relations variées depuis le commensalisme jusqu'à l'antagonisme, et le développement ou le maintien d'une espèce aux côtés d'une autre dépendra de son aptitude à la compétitivité : les organismes pionniers qui sont aptes à coloniser un bois non modifié sont souvent de faibles compétiteurs qui ne peuvent s'établir dans des tissus déjà envahis par d'autres espèces et qui, s'ils sont déjà installés, disparaissent rapidement. Cette disparition des pionniers, tout comme celle des espèces intervenant par la suite dans une succession parasitaire donnée, résulte d'un antagonisme entre les partenaires présents, les suivants éliminant vraisemblablement les précédents par des moyens tels que l'antibiose ou le mycoparasitisme. C'est ainsi que le Ceratocystis fimbriata, espèce pionnière pathogène chez le platane où il provoque la maladie du chancre coloré, ne se maintient dans la partie aérienne de l'arbre que pendant une durée limitée (un à deux ans environ); in vitro, nous avons pu montrer que ce parasite peut être détruit par plusieurs espèces de basidiomycètes lignivores (Fomes fomentarius, Lentinus tigrinus, Bjerkandera adusta, etc) que l'on retrouve fréquemment sur les arbres morts du chancre coloré (Grosclaude et al, 1990).

On a tenté de classer les champignons lignicoles en différentes catégories selon leur pouvoir pathogène, d'une part, et selon leurs aptitudes à la compétition à l'égard d'une microflore saprophyte déjà installée, d'autre part. Cette aptitude à la compétition a reçu le nom de kratovirulence (du grec kratein, dominer) ; elle rend compte en effet d'une "virulence de dominance» à l'égard d'organismes étrangers, et d'une plus ou moins grande résistance à la succession (Gramss, 1985). Globalement, chez les espèces lignicoles, le pouvoir pathogène varierait en sens inverse de la kratovirulence. Ainsi, les pionniers intervenant en début de succession auraient une faible kratovirulence et seraient donc facilement remplacés par d'autres espèces à l'égard desquelles ils ne sont pas compétitifs. À l'inverse, en fin de suc- cession, on trouverait des organismes à fort pouvoir compétitif, capables de s'installer sur des substrats déjà colonisés par une microflore qu'ils élimineront.

L'origine de ces communautés fongiques présentes dans l'arbre à un moment donné doit maintenant être envisagée. Si l'on peut s'accorder pour penser que la plupart des colonies présentes dérivent de spores ayant pénétré par des blessures, cette pénétration est le fait de toutes les espèces, à peu près simultanément pour une porte d'entrée donnée : à ce stade, on ne peut donc parler de succession parasitaire. La sélection ultérieure qui s'opérera sous l'influence des conditions de milieu existantes pourra alors favoriser telle espèce plutôt que telle autre, les premières à se développer étant les espèces pionnières, et la succession que l'on observera alors sera donc une succession de développement (et non de pénétration) des communautés fongiques. II faudra donc envisager que, dans les premiers stades d'altération du bois au moins, à côté de micro-organismes actifs, se trouvent masquées d'autres espèces en attente, ce qui constitue un état d'infection latente. Les exemples d'infection latente sont nombreux en pathologie végétale (Grosclaude, 1966a) et ils s'avèrent particulièrement intéressants lorsqu'ils concernent du bois sain en apparence : l'aération ou la teneur en eau de ce bois constituerait alors souvent le facteur déterminant l'inhibition ou le développement des espèces présentes (Chapela et Boddy, 1988).

La sélection qui va s'opérer tout au cours du temps, sélection due aux conditions de milieu régnant dans le bois et aux relations de compétition ou d'antagonisme entre les partenaires présents, aboutira généralement, à un moment donné, à la présence dominante d'un champignon lignivore particulier : celui qui aura su s'accomoder de toutes les contraintes existantes et qui, bien qu'étant le dernier à se développer, pourra provoquer des dommages graves.

Les champignons lignivores, qui donc apparaissent au terme des successions évoquées plus haut, peuvent être classés selon leur spécificité pour tel hôte ou groupe d'hôtes. Ainsi, si une très grande polyphagie est le fait d'Ungulina annosa, en revanche Fistulina hepatica ne s'observe que sur les feuillus. Plus spécialisé encore est Ungulina officinalis, hebergé seulement par mélèzes, cèdres et sapins Douglas, tandis qu'Ungulina betulina est inféodé au seul bouleau, et Mucidula mucida au hêtre. 


\section{RÉPONSE DE L'ARBRE AUX AGRESSIONS}

Les colorations anormales dont il a été question plus haut traduisent la réaction de l'arbre aux agressions.

Une observation attentive et une étude microbiologique simultanée permettront, sur la coupe transversale d'une branche au voisinage d'une blessure ancienne, de distinguer depuis la périphérie (aubier) jusqu'au centre :

- une zone de transition faiblement colorée, moins riche en eau que l'aubier et généralement stérile. La coloration observée serait la conséquence directe de la blessure (entrée d'air, etc). Puis, attenant à cette zone de transition, se situe :

- une zone de réaction, souvent assez intensément colorée en brun par des composés phénoliques (Shain, 1967), zone qui peut aussi être le siège de phénomènes de subérification (Biggs, 1987 ; Pearce, 1990). Dans cette zone, pourront être isolés différents micro-organismes pionniers, champignons ou bactéries.

Enfin, plus profondément encore se trouve une zone de bois dégradé, colonisé par les organismes lignivores (basidiomycètes supérieurs = hyménomycètes) (Shain, 1979). Selon l'état d'avancement et le type de pourriture, le bois sera plus ou moins décoloré et sa texture altérée. C'est à ce dernier niveau que s'observera la carie préludant à la formation de cavités plus ou moins importantes (tableau II).

C'est Shain qui, le premier, proposa les termes de zone de réaction pour désigner des régions du bois enrichies en substances phénoliques et intensément colorées en brun ou en noir, et constituant selon cet auteur la réponse non spécifique de l'hôte aux agressions subies (Shain, 1967).

Le bois de la zone de réaction est fortement coloré en brun, et non pas décoloré selon la traduction erronée faite parfois du qualificatif discoloured employé dans la littérature anglosaxonne. L'expression «zone de réaction» doit donc se substituer à celle de "bois de cœur pathologique" jadis employée, puisque cette zone se forme dans l'aubier et que sa teinte diffère notablement de celle du bois du cœur normal.

L'ensemble de ces différentes zones altérées revêt une forme caractéristique, toujours semblable dans la mesure où elles n'ont qu'une seule blessure pour origine. C'est ainsi qu'en coupe transversale on observera, pour une blessure latérale, une altération en forme de V, correspondant dans le plan longitudinal à une colonne allongée en fuseau. Lorsqu'il s'agit d'une blessure due à la section transversale d'une branche, les tissus altérés prennent la forme d'un cône renversé (fig 6). Cet aspect est la conséquence du développement plus rapide des micro-organismes dans le bois le plus ancien et donc le moins réactif.

Chronologiquement, le processus de dégradation du bois à la suite d'une blessure se déroulerait donc schématiquement en trois phases (Shigo et Sharon, 1968) :

- réaction de l'arbre au traumatisme physique produit par la blessure, se traduisant éventuellement par une coloration anormale du bois (zone de transition);

- colonisation par des organismes pionniers auxquels l'arbre réagit par la formation d'une zone de réaction intensément colorée ;

Tableau II. Aspect du bois en coupe transversale (on suppose qu'il existe une blessure transversale située plus haut).

Écorce

Moelle

\begin{tabular}{|c|c|c|c|c|}
\hline & Aubier & Zone de transition & Zone de réaction & Bois dégradé \\
\hline Teneur en eau & Humide & Peu humide & & \\
\hline Couleur & & Peu colorée & Très colorée & Décolorée \\
\hline Texture & & & & Fibreuse ou autre \\
\hline $\begin{array}{l}\text { Présence de } \\
\text { micro-organismes }\end{array}$ & & Stérile & $\begin{array}{l}\text { Pionniers: } \\
\text { bactéries } \\
\text { champignons divers }\end{array}$ & Basidiomycètes lignivores \\
\hline
\end{tabular}




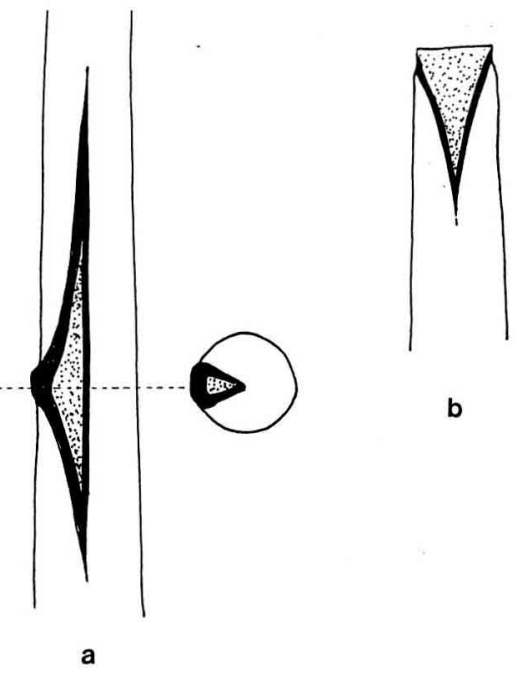

Fig 6. Aspect schématique du bois au niveau d'une blessure ancienne montrant la zone de réaction entourant la zone de bois dégradé. a. Après la section d'une branche latérale. $\mathbf{b}$. Après la section transversale d'une branche.

- colonisation, enfin, par des basidiomycètes lignivores générateurs de pourritures diverses (carie) : c'est la zone de bois dégradé.

Et, naturellement, la zone de bois dégradé pourra ou non s'accroître avec le temps, aux dépens de la zone de réaction, cette dernière gagnant à son tour du terrain au dépens de la zone de transition.

Si l'on observe maintement ce qui se passe dans les trois dimensions, l'étude de la réaction de l'arbre aux agressions que constituent les blessures et l'invasion par les micro-organismes a permis d'élaborer le concept de compartimentation dû à Shigo (Compartmentalization of Decay In Trees = CODIT. Shigo et Marx, 1977 ; Shigo, 1984). Selon ce concept, l'arbre dans lequel un micro-organisme s'est installé tend, par un processus de défense active, à isoler ce dernier dans un volume bien délimité. Ce volume est comparable aux compartiments étanches de la cale d'un navire, compartiments destinés, lors d'une avarie de la coque, à empêcher la pénétration de l'eau dans tout le bâtiment. Dans l'arbre, ces compartiments sont délimités par des "cloisons" agissant comme des barrières physiques (thyllose, gommose, subérisation) ou chimiques (fig 7). Les cloisons 1 sont constituées de 2 plans transversaux, parallèles entre eux, dans lesquels les vaisseaux vont être obturés (thyllose, gommose). La cloison 2 se constitue dans le plan tangentiel au niveau des anneaux de croissance, du côté interne. Les cloisons 3 se constituent dans les rayons ligneux (plan radial). La cloison 4 est l'homologue de la cloison 2 du

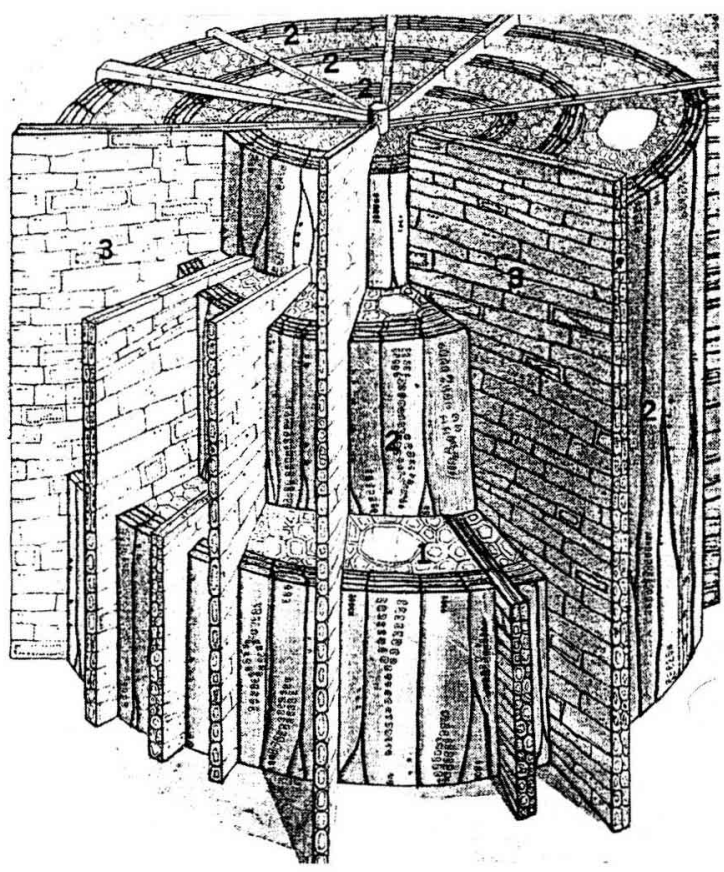

Fig 7. Schéma indiquant les positions des diverses cloisons (d'après Shigo et Marx, 1977). Cloisons 1 : situées dans 2 plans transversaux. À ce niveau, les vaisseaux sont obturés (une cloison similaire, parallèle à celle indiquée et située plus haut, n'a pas été figurée, afin de ne pas surcharger le dessin). Cloison 2 : située dans un plan tangentiel aux anneaux de croissance. Cloisons 3 : situées dans des plans radiaux au niveau des rayons ligneux. Cloison 4 : afin de ne pas surcharger le dessin, cette cloison n'est pas indiquée ici. Elle est parallèle à la cloison 2, mais située au niveau d'un anneau de croissance plus jeune (donc plus extérieur) que l'anneau de la cloison 2.

côté externe, mais c'est un tissu particulier élaboré par le cambium à proximité de la blessure et séparant les altérations du bois existant au moment de la blessure et les parties saines qui se formeront après.

Dans ces cloisons, outre la formation de thylles ou de gommes, la réaction de l'arbre se traduit par un dépôt de substances phénoliques, donc par une coloration bien visible. Ces cloisons constituent donc autant d'obstacles limitant ou empêchant la progression fongique. Mais la rapidité d'élaboration de ces cloisons et leur efficacité sont différentes: ainsi, selon Shigo (loc cit), la cloison 1 serait la plus faible, la moins efficace, à l'inverse de la cloison 4 . Les cloisons 1 à 3 des compartiments de Shigo, avec leurs limites diffuses, élaborées à partir du bois normal, pourraient correspondre aux zones de réaction dont il a été question plus haut - zones qui se déplaceraient au fur et à mesure de la progression des organismes pionniers. En revanche, la cloison 4, tout en faisant partie de la zone de ré- 
action au sens large, serait une véritable barrière marquée par des limites très nettes (Shain, 1979) (tableau II). Cette barrière, élaborée à partir du cambium, peut, à l'examen, révéler une structure particulière. En effet, on peut, chez certaines espèces, y observer la présence, entre les rayons ligneux, de lacunes à l'emplacement même où devraient se trouver des vaisseaux. Ces lacunes, disposées en un ou plusieurs cercles concentriques aux cernes d'accroissements, ont été décrites avec précision depuis fort longtemps chez les arbres fruitiers où elles ont parfois été appelées cavernes de résorption. Chez ces arbres, les lacunes se remplissent de gommes (Trecul, 1862 ; Prillieux, 1875 ; Ceruti et Scurti, 1953; Grosclaude, 1966b) (fig 8). Chez les gymnospermes, l'équivalent de ces lacunes gommeuses serait constitué par des canaux résinifères (Tippett et Shigo, 1981).

La théorie de la compartimentation selon Shigo a donné lieu à différentes critiques. Le terme même, employé par Shigo, de wall (cloison), parfois trop librement traduit par «barrière», suggère un obstacle infranchissable par les micro-organismes, ce qui trahit la pensée de l'auteur, celui-ci ayant affecté aux cloisons des numéros dans l'ordre croissant de leur pouvoir de résistance supposé vis-à-vis des agressions. En fait, la résistance variable des différentes cloisons découle en premier lieu de l'anatomie des zones concernées: ainsi, la voie

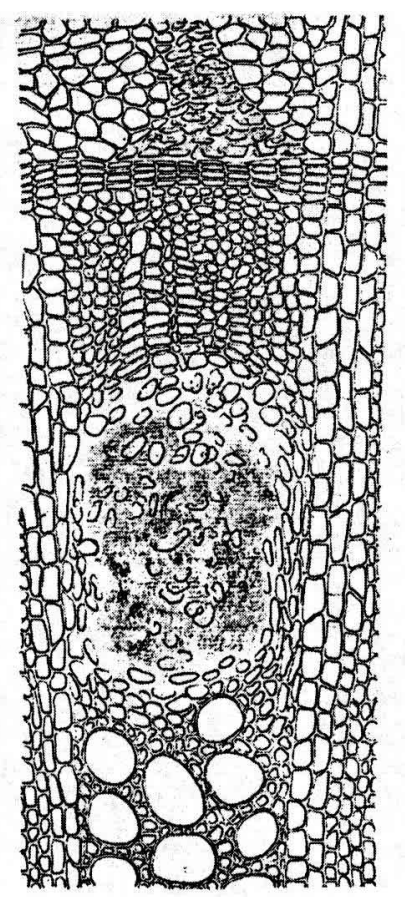

Fig 8. Lacune gommeuse chez le cerisier (d'après Ceruti et Scurti, 1953). axiale de pénétration des micro-organismes par les vaisseaux, étant a priori plus facile qu'une autre, a pour corollaire la plus grande "faiblesse" de la cloison 1 par exemple. On a par ailleurs fait remarquer que l'efficacité relative de ces cloisons n'était qu'indirecte, la conséquence de la compartimentation étant avant tout de recréer un environnement défavorable (car anoxique) aux champignons envahisseurs, tel qu'il était, avant l'introduction de l'air par une blessure (Rayner et Boddy, 1988). II faut donc considérer qu'aucune des cloisons définies par Shigo ne constitue une barrière infranchissable et que chacune d'entre elles peut posséder à cet égard des aptitudes différentes selon les champignons en présence. Ainsi, dans le cas de l'esca de la vigne, déjà évoqué, les cloisons 2 et $3 \mathrm{em}$ pêcheraient la progression de Phellinus $s p$ mais retarderaient seulement celle de Stereum hirsutum; quant à Cephalosporium, impliqué comme pionnier dans la succession, il serait capable de franchir toutes les cloisons, empêchant parfois même leur formation (Larignon, 1991).

D'autre part, l'observation de coupes dans un organe attaqué par des champignons lignivores montre bien souvent, dans le bois envahi, des reliquats de zones de réaction successives séparées les unes des autres par des plages peu colorées de tissu ligneux colonisé par ces champignons. D'où la conclusion que la progression fongique serait un phénomène discontinu marqué par des périodes d'arrêt durant lesquelles s'élaborerait une nouvelle zone de réaction au niveau du front d'avance du champignon. Ce qui implique donc que les zones de réaction, étant susceptibles d'être franchies par les champignons lignivores, ne constituent pas des barrières permanentes à la progression fongique (Pearce, 1991). Ces conclusions, étayées par l'analyse de certains des composants des zones de réaction, rejoignent celles énoncées longtemps auparavant par un chercheur hollandais qui, étudiant la maladie du plomb des arbres fruitiers provoquée par Chondrostereum purpureum, suggérait que les zones sombres observées dans le bois des arbres malades étaient la conséquence - et non la cause - d'un arrêt de croissance du champignon (Grosjean, 1955).

Si donc aucune barrière ou zone de réaction ne s'avère jamais infranchissable, on assistera tôt ou tard au dépérissement du sujet - ou de l'organe - envahi. Tout se passe en effet comme s'il existait une course de vitesse entre l'arbre et ses agresseurs : les champignons lignivores progressant depuis le bois central vers la périphérie, 
tandis que l'arbre forme, à partir du cambium, de nouvelle couches de bois sain. Mais on sait, par expérience, que la croissance d'un arbre, importante dans sa jeunesse, diminue graduellement avec l'âge: la sénescence se traduit donc, entre autres, par le fait que le bois sain nouvellement formé ne compense plus le bois plus ancien envahi par les champignons, la présence de ces derniers pouvant accentuer encore le ralentissement de la croissance.

\section{MESURES VISANT À LIMITER LE DÉVELOPPEMENT DES CHAMPIGNONS LIGNIVORES}

\section{"Le temps creuse le cœur des arbres comme il creuse le visage de l'homme." \\ Un conteur.}

À la fin de cette étude, il nous faut maintenant essayer d'en dégager quelques conseils pratiques. S'il paraît illusoire de vouloir arrêter le développement des champignons lignivores lorsque ceux-ci sont déjà bien installés dans l'arbre (et cela en dépit des tentatives de "chirurgie arboricole»), du moins peut-on, par des mesures préventives, éviter les dégâts dus à ces microorganismes?

La protection chimique des blessures par application d'un enduit fongicide sur les blessures fraîches, pratique souvent adoptée, va, pour un temps plus ou moins long, fermer la porte aux micro-organismes présents dans l'atmosphère; elle va donc permettre, dans l'immédiat, d'éviter la pénétration puis le développement de la plupart des micro-organismes pionniers parmi lesquels existent, nous le savons, de redoutables pathogènes (Ceratocystis fimbriata, Chondrostereum purpureum, Eutypa lata). L'expérience confirme le bien-fondé de ce qui vient d'être dit (Grosclaude, 1984). Cependant, la protection exercée par l'enduit fongicide n'est jamais totale et des pionniers s'établiront tout de même dans le tissu ligneux. La protection partielle réalisée à l'encontre de ce groupe de micro-organismes modifiera-t-elle, et de quelle façon, la séquence des événements et notamment le développement des champignons lignivores? Nous n'avons actuellement aucune réponse à cette question.

La protection conférée par l'enduit fongicide a par ailleurs une durée limitée: le revêtement s'écaille, la matière active elle-même peut, à la longue, se dégrader. Le tissu ligneux va donc une nouvelle fois se trouver exposé aux agents extérieurs. Mais il s'agira alors de blessures anciennes et par conséquent d'un tissu ligneux fortement modifié par la présence de thyllose ou de gommose, et en outre déjà colonisé par les pionniers ayant échappé au premier traitement. De nouvelles infections vont donc pouvoir se produire, qui seront alors le fait des envahisseurs séquentiels, et notamment des champignons lignivores (toutefois, nous avons déjà souligné le fait que les blessures âgées, ne pouvant aspirer les spores, constituent des sites peu favorables au développement de ces dernières). Contre de telles infections, nous sommes donc à peu près désarmés, à moins de s'astreindre au renouvellement périodique de l'enduit fongicide appliqué, ce qui est en pratique très difficile à réaliser.

La protection biologique des blessures par l'apport d'un agent antagoniste, champignon ou bactérie, peut également être envisagée. Toutefois, plus spécifique que la protection chimique, elle nécessite de choisir l'antagoniste susceptible d'agir contre le pathogène visé. Or on ne connaît actuellement que peu d'exemples concluants à ce sujet, excepté le cas du Trichoderma harzianum employé pour lutter contre le Chondrostereum purpureum des arbres fruitiers (Grosclaude, 1973). En revanche, la protection biologique pourrait s'avérer plus durable que la protection chimique puisque l'agent biologique, élément vivant, pourra se multiplier dans le tissu ligneux, alors que le produit chimique ira en se dégradant au cours du temps.

Mais protection chimique ou protection biologique ne concernent que les blessures visibles. Or, sans parler des blessures accidentelles ou bien de trop petites dimensions, qui peuvent échapper longtemps à notre attention, nous avons déjà signalé le phénomène de l'élagage naturel, qui peut créer des voies d'entrée pour certaines espèces lignicoles, voies d'entrée qui ne sont pas justiciables d'un traitement quel qu'il soit.

On veillera donc, en définitive, à éviter toute blessure inutile aux arbres en limitant la taille à des branches de petit diamètre, la cicatrisation (qui est le recouvrement de la plaie par des tissus néoformés et non pas, comme on le pense souvent, sa perte de réceptivité aux parasites) s'effectuant plus rapidement pour des plaies de petit diamètre (Neely, 1988). C'est d'ailleurs à partir de la fin du repos végétatif que peut commencer le processus de cicatrisation : c'est donc à cette époque qu'il semble judicieux d'entre- 
prendre les opérations de taille (de ce point de vue, une taille en vert, c'est-à-dire pratiquée en pleine végétation, serait également admissible, mais outre le fait qu'elle présente des difficultés d'ordre pratique, on ne peut affirmer qu'elle ne soit pas néfaste sur le plan physiologique). En outre, la taille des branches devra respecter certaines règles, en particulier la coupe effectuée ne devra pas laisser de chicot : celui-ci empêche en effet le recouvrement par le bourrelet cicatriciel et, de plus, constitué de tissus morts, il peut être un foyer d'infection. À l'opposé, il ne faudra pas réaliser une coupe rase : il semble en effet, comme on a pu le démontrer dans quelques cas particuliers, que la meilleure solution soit de conserver le "collet" de la branche coupée, car c'est là que s'élaboreraient les barrières limitant la progression des micro-organismes (Biggs, 1989) (fig 9). Chez les espèces à grand développement, la taille dite "radicale", c'est-à-dire mutilante et traumatisante, dont les plaies ne cicatrisent jamais entièrement sinon au bout d'un temps très long, sera donc prohibée au profit des tailles douces dites «au grimper». L'application d'un enduit fongicide (ou d'un antagoniste dans le cas de la protection biologique) sera effectuée immédiatement; le renouvellement de l'opération sera inutile si la cicatrisation s'effectue rapidement. Enfin, un élagage artificiel conduit selon ces principes pourrait probablement limiter l'élagage naturel dont les conséquences évoquées plus haut semblent difficiles à éviter.

\section{CONCLUSION}

Comme nous venons de le montrer, nous ne disposons pas actuellement de moyens qui nous permettraient d'éviter totalement la pénétration et le développement d'organismes lignicoles. II existera toujours des blessures non protégées chez un arbre, et la protection appliquée aux
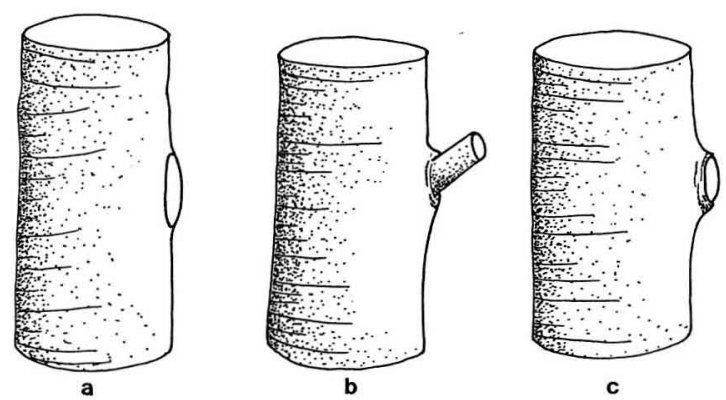

Fig 9. Section d'une branche latérale: a. Coupe rase : b. Coupe avec chicot : c. Coupe conservant le "collet" de la branche. autres ne sera jamais totalement efficace ni suffisamment durable. Quant à l'élagage naturel, il sera toujours difficile, sinon impossible à limiter. Dans le meilleur des cas, nous parviendrons donc seulement à retarder le développement des champignons lignivores. Mais il faut se faire une raison : les arbres creux servent d'abri à de nombreuses espèces animales qui ont un rôle dans l'équilibre général, et le développement de ces champignons lignivores doit, quant à lui, être considéré comme le processus nécessaire conduisant à la «mort naturelle» d'un individu et à la poursuite du cycle biologique.

L'arbre, comme tout être vivant, n'est pas éternel, mais il nous revient de lui éviter une mort prématurée, conséquence de nos erreurs et, d'autre part, de prévoir son remplacement lorsqu'il aura atteint un âge avancé. Nous permettrat-on alors d'appliquer aux arbres sur le déclin ces phrases de Jean Guitton: «Dans ces épreuves, je suis partagé entre deux sentiments contraires : la mélancolie en voyant s'abîmer ce que j'avais aimé. Mais aussi l'espérance: lorsque les éléments sont décomposés, ils peuvent se recomposer d'une manière plus pure, et préparer une nouvelle aurore.»

\section{RÉFÉRENCES}

Anonyme (1964) Multilingual glossary of terms used in wood anatomy. Inst suisse de recherches forestières, mémoires, vol 40

Anselmi N (1990) Wood deterioration in poplars following decline after water stress. Eur J For Pathol 20, 321-328

Von Aufsess H (1975) Über die Bildung einer Schutzsperre an der Astbasis von Laub- und Nadelbäumen und ihre Wirksamkeit gegen das Eindringen von Pilzen in das Kernholz lebender Bäume. Forstw Cb/94, 140-152

Beckman CH, Keller JL (1977) Vessels do end. Letter to editor. Phytopathology 67, 954-956

Biggs AR (1987) Occurrence and location of suberin in wound reaction zones in xylem of 17 tree species. Phytopathology 77, 718-725

Biggs AR (1989) Effect of pruning technique on Leucostoma infection and callus formation over wounds in peach trees. Plant Dis 73, 771-773

Blanchette A (1991) Delignification by wood-decay fungi. Annu Rev Phytopathol 29, 381-398

Boddy L, Rayner ADM (1983) Origins of decay in living deciduous trees: the role of moisture content and reappraisal of the expanded concept of tree decay. New Phytol 94, 623-641

Bonsen KJM, Bucher HP (1991) What arborists have to know about vessel plugs. Arbor J 15, 13-17 
Butin H, Kowalski T (1983) Die natürliche Astreinigung und ihre biologischen Voraussetzungen. I. Die Pilzflora der Buche (Fagus sylvatica L). Eur J For Pathol 13, 322-334

Butin H, Kowalski T (1986) Die natürliche Astreinigung und ihre biologischen Voraussetzungen. III. Die Pilzflora von Ahorn, Erle, Birke, Hainbuche un Esche. Eur J For Pathol 16, 129-138

Butin H, Kowalski T (1990) Die natürliche Astreinigung und ihre biologischen Voraussetzungen. V. Die Pilzflora von Fichte, Kiefer und Lärche. Eur J For Pathol $20,44-54$

Catesson AM, Czaninski $Y$, Peresse M, Moreau M (1976) Sécrétions intravasculaires de substances "gommeuses" par les cellules associées aux vaisseaux en réaction à une attaque parasitaire. Soc Bot Coll Sécret Véget 93-107

Ceruti A, Scurti J (1953) Sulla formazione delle gomme nel ciliegio (Prunus avium L) Nota I: Morfologia. Ann Sper Agrar 489-499

Chaney WR (1979) Leaf and twig abscission relationships in a mature white oak. Can J For Res 9, 345348

Chapela IH, Boddy L (1988) Fungal colonization of attached beech branches. 2. Spatial and temporal organization of communities arising from latent invaders in bark and functional sapwood, under different moisture regimes. New Phytol 110, 47-57

Chattaway MM (1949) The development of tyloses and secretion of gum in heartwood formation. Aust $\mathrm{J} \mathrm{Sci}$ Res B 2, 227-240

Christensen $O$ (1975) Wood litter fall in relation to abscission, environmental factors, and the decomposition cycle in a Danish oak forest. Oikos 26, 187195

Cruiziat P, Tyree MT (1990) La montée de la sève dans les arbres. Recherche 21, 406-414

D'Aeth HRX (1939) A survey of interaction between fungi. Biol Rev 14, 105-131

Dixon HH (1924) The transpiration stram. Univ London Press, Londres

Gramss G (1985) Invasion of wood by basidiomycetous fungi. I. Pathosism and saprophytism as determined by certain experimentally accessible virulence properties. J Basic Microbiol 25, 305-324

Grosclaude C (1966a) Champignons parasites latents chez les arbres. Rev Zool Agric Appl 4-6, 82-91

Grosclaude C (1966b) La gommose des arbres fruitiers. Ann Epiphyt 17, 129-137

Grosclaude C (1973) Protection biologique des plaies de taille vis-à-vis du Stereum purpureum, agent du «plomb» des arbres fruitiers. Rev Suisse Vitic Arboric Hortic 5, 51-53

Grosclaude C (1974) La pénétration des spores de champignons dans les blessures de taille des arbres fruitiers. Application au cas de la protection biologique vis-à-vis du Stereum purpureum. Rev Zool Agric Pathol Vég 1, 1-21
Grosclaude C (1984) L'efficacité des produits de protection des blessures chez les végétaux ligneux. Phytoma 362, 38-42

Grosclaude C, Olivier R, Romiti C, Pizzuto JC (1990) Action antagoniste, sur bois in vitro, de quelques basidiomycètes lignicoles vis-à-vis du Ceratocystis fimbriata $f$ platani présent dans le tissu ligneux. agronomie 10, 403-405

Grosjean J (1955) Annual periodicity in the parasitical activity of Stereum purpureum. T PI Ziekten 62, 226-235 (en néerlandais)

Hartig R (1878) Die Zersetzungserscheinungen des Holzes der Nadelholzbaume und der Eiche in forstlicher, botanischer und chemischer Richtung. Springer Verlag, Berlin

Hintikka V (1973) Passive entry of fungus spores into wood. Karstenia 13, 5-8

Huguet JG (1973) Étude des facteurs modifiant la composition de la sève de printemps des arbres fruitiers. Ann Agron 24, 477-501

Larignon P (1991) Contribution à l'identification et au mode d'action des champignons associés au syndrome de l'esca de la vigne. Thèse Univ Bordeaux II

Mer E (1887) Recherche sur la formation du bois parfait chez les essences feuillues. Bull Soc Bot Fr 34 , 341-363

Neely Dan (1988) Tree wound closure. J Arboric 14, 148-152

Nepveu G (1991) L'eau dans le bois de l'arbre sur pied et dans le bois mis en œuvre. $I n$ : Colloque : L'eau dans l'arbre et le bois. INRA, Champenoux

Paillassa E, Dubos B, Larignon P, Chevrier JC (1992) L'inoculum infectieux d'Eutypa lata. Sa formation, son évolution et sa dispersion : les facteurs qui les influencent. Phytoma 440, 21-23

Pearce RB (1990) Occurence of decay-associated xylem suberization in a range of woody species. Eur $J$ For Pathol 20, 275-289

Pearce RB (1991) Reaction zone relics and the dynamics of fungal spread in the xylem of woody angiosperms. Physiol Mol Plant Pathol 39, 41-55

Prillieux E (1875) Étude sur la formation de la gomme dans les arbres fruitiers. Ann Sci Nat (Bot) Sér 6, 176-200

Rameau JC, Mansion D, Dume G (1989) Flore forestière française. Guide écologique illustré. 1. Plaines et collines. Institut pour le développement forestier, Paris, $1783 p$

Ravaz L (1909) Sur l'apoplexie de la vigne. Prog Agric Vitic 52, 574-579

Rayner ADM (1985) Water and the origins of decay in trees. In : Symposium of Lancaster: water, fungi and plants. 321-342

Rayner ADM, Boddy L (1988) Fungal decomposition of wood. John Wiley \& Sons, Chichester, $587 \mathrm{pp}$

Salleo S, Logullo MA, Siracusano L (1984) Distribution of vessel ends in stems of some diffuse- and 
ring-porous trees: the nodal regions as "safety zones" of the water conducting system. Ann Bot $54,543-552$

Shain L (1967) Resistance of sapwood in stems of loblolly pine to infection by Fomes annosus. Phytopathology 57, 1034-1045

Shain L (1979) Dynamic responses of differentiated sapwood to injury and infection. Phytopathology 69, 1143-1147

Scheffer TC, Cowling EB (1966) Natural resistance of wood to microbial deterioration. Annu Rev Phytopathol 4, 147-170

Shigo AL (1967) Successions of organisms in discoloration and decay of wood. Int Rev For Res 2, 237299

Shigo AL (1984) Compartmentalization: a conceptual framework for understanding how trees grow and defend themselves. Annu Rev Phytopathol 22, 189214

Shigo AL, Marx HG (1977) Compartmentalization of decay in trees. US dept agric, for serv, agric inf bull 405
Shigo AL, Sharon EM (1968) Discoloration and decay in hardwoods following inoculations with hymenomycetes. Phytopathology 58, 1493-1498

Thomas M (1933) The natural abscission of twigs in Canadian black poplar (Populus serotina Hartig). Naturalist April, 79-86

Tippett JT, Shigo AL (1981) Barriers to decay in conifer roots. Eur J For Pathol 11, 51-59

Trecul A (1862) Production de la gomme chez le cerisier, le prunier, l'amandier, l'abricotier et le pêcher. PV Séances Soc Philomat 82-90

Vandermolen GE, Beckman CH, Rodehorst E (1977) Vascular gelation: a general response phenomenon following infection. Physiol Plant Pathol 11, 95-100

Wall RE, Kuntz JE (1964) Water-soluble substances in dead branches of aspen (Populus tremuloides Mich $\mathrm{x}$ ) and their effects on Fomes igniarius. Can $J$ Bot 42, 969-977

Zimmermann $\mathrm{MH}$ (1983) Xylem structure and the ascent of sap. Springer Verlag, Berlin $141 \mathrm{p}$

Zimmermann MH, Brown CL (1974) Tree structure and function. Springer Verlag, Berlin $331 \mathrm{p}$ 